\title{
Determinants, incidence and perinatal outcomes of multiple pregnancy deliveries in a low-resource setting, Mpilo Central Hospital, Bulawayo, Zimbabwe
}

\begin{abstract}
Background: Multiple pregnancies are high risk pregnancies compared to singletons They may result in poor feto-maternal outcomes. Traditionally, these pregnancies are associated with anaemia, preeclampsia, preterm deliveries and postpartum haemorrhage. In low-resource settings, these women and their babies may face increased risks of poor perinatal outcomes. The objective of this study was to document for the first time the determinants, incidence and perinatal outcomes of multiple pregnancies for Mpilo Central Hospital.

Methods: This was a retrospective descriptive study covering the period between 1 January 2017 and 31 December 2017 in a tertiary teaching hospital. A paper data collection sheet was used to collect the information. All twin/triplet deliveries $>24$ weeks gestation born at the labour ward were included in the study. The data was then analysed.

Results: The incidence of multiple pregnancy at Mpilo Central Hospital was $1.7 \%$. The 20-25 year old age group had the highest percentage at $25.5 \%$. Nulliparous women had the highest percentage at $28.4 \%$ of the patients. Booked/referred patients constituted the majority at $45.4 \%$, followed by instutional booked at $39.0 \% .37 .5 \%$ of the pregnancies were preterm $(<36+6$ weeks gestation). The majority $(97.2 \%)$ had no postnatal complications. $2.8 \%$ had postpartum haemorrhage. There was a statistical difference between the two groups, $\mathrm{P}=0.05$. There were no maternal deaths. $93.2 \%$ were live births and $6.8 \%$ were stillbirths, with a statistical difference between the two groups, $\mathrm{P}=0.05$. Female/female twins were $28.4 \%$, male $/$ male $26.9 \%$ and the majority being mixed at $44.7 \%$. The majority $(81.6 \%)$ had good 1 minute Apgar scores $>7$ and $18.4 \%$ had 1 minute Apgar scores $<7$, with a statistical difference between the two groups, $\mathrm{P}=0.01$. By 5 minutes, a vast percentage $92.3 \%$ had Apgar scores $>7$ and only $7.7 \%$ had Apgar scores $<7$, with a statistical difference between the two groups, $\mathrm{P}=0.05$.

Conclusion: The incidence of multiple pregnancy at Mpilo Central Hospital was $1.7 \%$. The major determinants of multiple pregnancies were the 20-25 year old age group, nulliparity and being $<36+6$ weeks of gestation. The majority of women had no postnatal complications and there were no maternal deaths. The majority of babies were live births with good 1 minute Apgar scores $>7$. The babies were of good birth weights $(2501-3000 \mathrm{~g})$
\end{abstract}

Keywords: multiple pregnancy, determinants, incidence, perinatal, outcomes, lowresource, settings
Volume 8 Issue 2 - 2019

\author{
Solwayo Ngwenya \\ Department of Obstetrics and Gynecology, Mpilo Central \\ Hospital, Zimbabwe
}

Correspondence: Solwayo Ngwenya, Department of Obstetrics and Gynecology, Mpilo Central Hospital, P.O. Box 2096, Vera Road, Mzilikazi, Bulawayo, Matabeleland, Zimbabwe, Tel +2639214965, Email drsolngwe@yahoo.co.uk

Received: December 31, 2018 | Published: March 05, 2019
Abbreviations: ARV, antiretrovirials; HIV, human immuno deficiency virus; LSCS, lower segment caesarean section; SD, standard deviation

\section{Background}

Mpilo Central Hospital is located in Bulawayo and deliveries 9000 deliveries per year. Bulawayo is the second largest city in Zimbabwe after the capital city Harare, with a population of 653 , 337 as of the 2012 census. ${ }^{1}$ The unit receives referrals from urban and rural centres. It is located in Matabeleland, $439 \mathrm{~km}$ southwest of Harare, on the way to Victoria Falls. Multiple gestations are high risk pregnancies associated with significant poor perinatal outcomes compared to singletons. ${ }^{2}$ In low- and middle-income countries they pose a high risk to mothers due to inherent biological risks and scarcity of health resources. ${ }^{3}$ In low-resourced, Nigeria the incidence of twin gestation was found to be $2.3 \%$, the highest in the world. ${ }^{4,5}$ The second twin is at greater risk of adverse perinatal outcome than the first twin. ${ }^{6}$ The second twin could die or suffer brain damage due to delayed delivery. Women with multiple gestations should have prompt recourse to emergency caesarean section to avoid poor fetal outcomes. Complications of multiple gestations include anaemia, preeclampsia/eclampsia and preterm labour. The women are at risk of postpartum haemorrhage and death. The fetal complications include twin-twin transfusion syndrome in monochromic twins, congenital malformations, intrauterine death, complications of prematurity, locked twins and birth asphyxia. Women with such high risk pregnancies should book early; have an early ultrasound for dating, fetal number and chorionicity. They should book in a centre where there is comprehensive emergency obstetric care. In low-resource settings, women carry such high risk pregnancies without access to such resources. 


\section{Methods}

This was a retrospective descriptive study covering the period between 1 January 2017 and 31 December 2017 in a tertiary teaching hospital. A paper data collection sheet was used to collect the information. All twin/triplet deliveries $>24$ weeks gestation born at the labour ward were included in the study. The data was then analysed. The SPSS Version 21(IBM Corp., Armonk, NY, USA) statistical tool was used to calculate the mean and standard deviation (SD) figures. Descriptive statistics were used to examine patients' characteristics and the outcomes. Chi-square test was used to calculate P-values. A $\mathrm{P}$-value of $<0.05$ was considered statistically significant.

\section{Results}

There were 8439 live births at the unit the period between 1 January 2017 and 31 December 2017. There were 141 multiple pregnancies made up of 140 sets of twin pregnancies and 1 set of triplets. There were 283 babies born, 3 were born at home leaving 280 babies for analysis. The incidence of multiple pregnancy at Mpilo Central Hospital was $1.7 \%$. Tables $1-4$ show most of the results. The $20-25$ year old age group had the highest percentage at $25.5 \%$. Teenagers (16-19 year olds) accounted for $12.8 \%$ of the patients. After the age of 36 years, multiple pregnancy rates decline with those $>40$ years accounting for only $0.7 \%$. Nulliparous women had the highest percentage at $28.4 \%$ of the patients, followed by Para 1 at $25.5 \%$ and grandmultiparous women at $10.7 \%$. Booked/referred patients constituted the majority at $45.4 \%$, followed by instutional booked at $39.0 \%$. Unbooked cases were $15.6 \%$. HIV infected women were $19.9 \%$, the majority $(62.4 \%)$ were seronegative and $17.7 \%$ of the patients presented with unknown HIV status. $37.5 \%$ of the pregnancies were preterm $(<36+6$ weeks gestation), $30.6 \%$ were term(37-40 weeks gestation) and $6.4 \%$ were postdates( $>40$ weeks gestation). More than a quarter $(25.5 \%)$ was of unknown gestation. More than half $(50.5 \%)$ had no antenatal complications. Other complications included preeclampsia $(5.7 \%)$, abruption $(2.1 \%)$, retained twin $(2.1 \%)$, anaemia, hand prolapsed and footling breech all at $0.7 \%$ each (Table 1 ).

Most (57.4\%) were delivered by caesarean section for the leading breech twin and $2.1 \%$ for a retained twin. The commonest vaginal delivery was cephalic/cephalic (31.9\%), followed by breech/breech $(5.0 \%)$, cephalic/breech $(4.3 \%)$ and the least was breech/cephalic $(1.4 \%)$. There were no vacuum or forceps deliveries. The majority $(97.2 \%)$ had no postnatal complications and $2.8 \%$ had postpartum haemorrhage. There was a statistical difference between the two groups, $\mathrm{P}=0.05$. There were no cases of maternal deaths during the study period (Table 2 ).

Live births constituted $93.2 \%$ and $6.8 \%$ were stillbirths, with a statistical difference between the two groups, $\mathrm{P}=0.05$. Female/female twins were $28.4 \%$, male/male $26.9 \%$ and the majority being mixed at $44.7 \%$. The majority ( $81.6 \%$ ) had good 1 minute Apgar scores $>7$ and $18.4 \%$ had 1 minute Apgar scores $<7$, with a statistical difference between the two groups, $\mathrm{P}=0.01$. By 5 minutes, 92.3\% had Apgar scores $>7$ and only $7.7 \%$ had Apgar scores $<7$, with a statistical difference between the two groups, $\mathrm{P}=0.05$. There was 1 set of conjoined twins and these weighed $4500 \mathrm{~g}$ and 1 papyraceous twin that was not weighable, leaving 278 babies for birth weight analysis. The commonest birth weights range was 2501-3000g (28.1\%) (Table 3 \& Table 4).
Table I Maternal demographic details

\begin{tabular}{|c|c|c|}
\hline Characteristic & $N=|4|(\%)$ & $P$ value \\
\hline \multicolumn{3}{|l|}{ Age(years) } \\
\hline Dec-15 & $0(0 \%)$ & \\
\hline $16-19$ & $18(12.8 \%)$ & \\
\hline $20-25$ & $36(25.5 \%)$ & \\
\hline $26-30$ & $31(22.0 \%)$ & \\
\hline $31-35$ & $35(24.8 \%)$ & \\
\hline $36-40$ & $16(11.3 \%)$ & \\
\hline $41-45$ & $4(2.9 \%)$ & \\
\hline$>45$ & $\mathrm{I}(0.7 \%)$ & \\
\hline \multicolumn{3}{|l|}{ Parity } \\
\hline 0 & $40(28.4 \%)$ & \\
\hline I & $36(25.5 \%)$ & \\
\hline 2 & $25(17.7 \%)$ & \\
\hline 3 & $25(\mid 7.7 \%)$ & \\
\hline 4 & $9(6.4 \%)$ & \\
\hline 5 & $6(4.3 \%)$ & \\
\hline \multicolumn{3}{|c|}{ Gestational age(weeks) } \\
\hline$<28$ & $4(2.8 \%)$ & \\
\hline $28+1-33+6$ & $16(11.3 \%)$ & \\
\hline $34-36+6$ & $33(23.4 \%)$ & \\
\hline $37-40$ & $43(30.6 \%)$ & \\
\hline$>40$ & $9(6.4 \%)$ & \\
\hline Unknown & $36(25.5 \%)$ & \\
\hline
\end{tabular}

Table 2 Maternal characteristics

\begin{tabular}{lcl}
\hline Variable & N=141(\%) & P value \\
\hline Booking status & & \\
Booked $>4$ ANC visits & $55(39.0 \%)$ & \\
Booked(referral) & $64(45.4 \%)$ & \\
Unbooked & $22(15.6 \%)$ & 0.1 \\
HIV status & \\
HIV -ve & $88(62.4 \%)$ & \\
HIV +ve & $28(19.9 \%)$ & \\
Unknown & $25(17.7 \%)$ & 0.1 \\
ARV's & 28 \\
Antenatal complications None & \\
Preterm labour & $71(50.5 \%)$ \\
Preeclampsia & $53(37.5 \%)$ \\
Abruptio & $8(5.7 \%)$ \\
Retained twin & $3(2.1 \%)$ \\
Hand prolapse & $3(2.1 \%)$ \\
Anaemia & $1(0.7 \%)$ \\
Footling breech & $1(0.7 \%)$ \\
& $1(0.7 \%)$ & \\
\end{tabular}


Table 3 Mode of delivery and maternal complications

\begin{tabular}{lll}
\hline Variable & $\mathbf{N}=\mathbf{1 4 1} / \mathbf{( \% )}$ & P value \\
\hline Mode of delivery & \\
Vacuum/forceps & $0(0 \%)$ \\
LSCS(twin 1 breech) & $81(57.4 \%)$ \\
LSCS(retained twin) & $3(2.1 \%)$ \\
Vaginal(cephalic/cephalic) & $45(31.9 \%)$ \\
(breech/breech) & $7(5.0 \%)$ \\
(cephalic/breech) & $6(4.3 \%)$ \\
(breech/cephalic) & $2(1.4 \%)$ \\
Maternal complications & \\
None & $137(97.2 \%)$ \\
Postpartum haemorrhage & $4(2.8 \%)$ & 0.05 \\
Maternal death & $0(0 \%)$ & \\
\hline
\end{tabular}

Table 4 Fetal characteristics

\begin{tabular}{|c|c|c|}
\hline Variable & $\mathrm{N}=\mathbf{2 8 0} /(\%)$ & P value \\
\hline \multicolumn{3}{|l|}{ Outcome } \\
\hline Live births & $261(93.2 \%)$ & \\
\hline Stillbirths & $19(6.8 \%)$ & 0.05 \\
\hline \multicolumn{3}{|l|}{ Fetal sex } \\
\hline Female & $144(51.4 \%)$ & \\
\hline Male & $136(48.6 \%)$ & \\
\hline \multicolumn{3}{|l|}{ Combination types } \\
\hline Female/female & $40(28.4 \%)$ & \\
\hline Male/male & $38(26.9 \%)$ & \\
\hline Mixed & $63(44.7 \%)$ & \\
\hline \multicolumn{3}{|l|}{ Apgar scores } \\
\hline 1 minute $<7$ & $48(18.4 \%)$ & \\
\hline 1 minute $>7$ & $213(81.6 \%)$ & 0.01 \\
\hline 5 minute $<7$ & $20(7.7 \%)$ & \\
\hline 5 minute $>7$ & $241(92.9 \%)$ & 0.05 \\
\hline \multicolumn{3}{|l|}{ Birth weight $\mathrm{N}=278$} \\
\hline $500-1000 \mathrm{~g}$ & $18(6.4 \%)$ & \\
\hline $1001-1500 \mathrm{~g}$ & $21(7.6 \%)$ & \\
\hline $1501-2000 \mathrm{~g}$ & $60(21.6 \%)$ & \\
\hline $2001-2500 \mathrm{~g}$ & $78(28.1 \%)$ & \\
\hline $2501-3000 \mathrm{~g}$ & $81(29.1 \%)$ & \\
\hline $3001-3500 \mathrm{~g}$ & $19(6.8 \%)$ & \\
\hline $3501-4000 \mathrm{~g}$ & $0(0 \%)$ & \\
\hline$>4000 \mathrm{~g}$ conjoined & $1(0.4 \%)$ & \\
\hline
\end{tabular}

\section{Discussion}

The incidence of multiple pregnancy at Mpilo Central Hospital was found to be $1.7 \%$ lower than that reported elsewhere in Nigerian hospitals. ${ }^{4,5}$ The younger age groups $(<25$ years $)$ and nulliparity were associated with higher twinning rates than other groups. Older grandmultiparous women had lower rates. This is probably due to declining fertility rates with advancing age. A study in Nairobi, Kenya also had similar findings. ${ }^{7}$ Despite being high risk groups, referring centres seemed to have booked multiple gestations and referred them when complications arose. Some women were unbooked (15.6\%). This needs changing so that such women are all booked at the tertiary centre where facilities for emergency caesarean section are readily available.

The HIV seropositive rate was $19.9 \%$ and $17.7 \%$ being of unknown status. They could potentially result in babies being exposed to HIV infection. The prevalence of HIV infection amongst the obstetric patient in the unit is $16 \%$, the national figure for Zimbabwe is $15 \%$ amongst adults 15 years and above. ${ }^{8}$ The figure for HIV seropositive in multiple pregnancy deliveries was slightly higher than the unit and national average.

More than a third $(37.5 \%)$ were delivered premature $(<36+6$ weeks gestation) confirming the classical commonest association between multiple gestations and preterm delivery. ${ }^{9}$ There were $15.6 \%$ of unbooked patient which is a recurring theme in low-resourced countries, in a study in Nigeria the figure was as high as $65.7 \% .{ }^{10} \mathrm{~A}$ quarter of women had unknown gestation due to a combination of being unbooked and lack of access to ultrasonography.

Most women had caesarean section for the leading twin being breech and a retained second twin (total 59.5\%). The commonest vaginal delivery was cephalic/cephalic at $31.9 \%$ of the cases. The immediate postnatal complication was postpartum haemorrhage at $2.8 \%$. This is lower than that reported in a study in Nigeria where it was found to be $8.5 \% .^{11}$ There were no cases of maternal deaths recorded among the 141 women. There majority of fetal outcome were live births and minority were stillbirths, with a statistical difference between the two groups, $\mathrm{P}=0.05$. Therefore multiple pregnancy mainly ended in live births. There is lack of ultrasonography services and ultrasound scans for dating and determing chorionicity are not the routine. Women either present late for such testing or simply do not book but present in labour. The immediate perinatal period seemed to be encouraging with the majority of newborn infants having good 1 minute Apgar scores $>7$.

\section{Conclusions}

The incidence of multiple pregnancy at Mpilo Central Hospital was found to be $1.7 \%$ lower than that reported elsewhere in the literature. The younger age groups $(<25$ years) and nulliparity were associated with higher twinning rates than other groups. More than a third $(37.5 \%)$ were delivered premature $(<36+6$ weeks gestation). There were no cases of maternal deaths recorded among the studied women. The fetal outcome was good with the majority being live births. The immediate perinatal period seemed to be encouraging with the majority of newborn infants having good 1 minute Apgar scores $>7$. There is need to improve the referral system so that all multiple pregnancies are booked at a tertiary centre with recourse to prompt emergency caesarean section for complications. Due to limited resources, early ultrasound scans to determine chorionicity and screen for twin-twin transfusion syndrome remain difficult in low-resourced settings. 


\section{Declarations}

\section{Ethics approval and consent to participate}

The Ethics Committee at Mpilo Central Hospital gave a waiver for retrospective and non-intervention studies to go ahead in the institution as long as the data remained anonymous. No ethical issues arose during the study as all the data was anonymous. No patient consent was necessary. Minutes of the Committee's inaugural meeting held on the 13th October 2016 set out the requirements of all the studies at the institution.

\section{Acknowledgments}

None.

\section{Conflicts of interest}

The author declares there are no conflicts of interest.

\section{References}

1. Zimdat. Census Report 2012.

2. Olusanya BO. Perinatal outcomes of multiple births in southwest Nigeria. J Health Popul Nutr. 2011; 29(6):639-647.

3. Vogel JP, Torloni MR, Seuc A, et al. Maternal and perinatal outcomes of twin pregnancy in 23 low-and middle-income countries. PLoS One. 2013;8(8):e70549.
4. Kullima AA, Audu BM, Geidam AD. Outcome of twin deliveries at the University of Maiduguri Teaching Hospital: a 5 year review. Niger $J$ Clin Pract. 2011;14(3):345-348.

5. Akada GO, Agida TE, Onafowokan O, et al. Review of twin pregnancies in a tertiary hospital in Abuja, Nigeria. $J$ Health Popul Nutri. 2013;31(2):272-277.

6. Armson BA, O Connell C, Persad V. Determinants of perinatal mortality and serious neonatal morbidity in the second twin. Obstet Gynaecol. 2006; 108(3Pt 1):556-564.

7. Musili F, Karanja JG. Multifoetal pregnancies at a maternity hospital in Nairobi. East Afr Med J. 2009;86(4):162-165.

8. Zimdat. Zimbabwe Demographic Health Survey Report 2010/11. 470 p.

9. Aduloju OP, Olofinbiyi B, Olagbuji BN, et al. Obstetric outcome of twin gestations in a tertiary hospital South-western Nigeria. J Matern Fetal Neonatal Med. 2015;28(8):900-904.

10. Igberase GO, Ebeigbe PN, Bock Oruma A. Twinning rate in a rural mission tertiary hospital in the Niger delta, Nigeria. J Obstet Gynaecol. 2008;28(6):586-589.

11. Nwankwo TO, Aniebue UU, Ezenkwele E, et al. Pregnancy outcome and factors affecting vaginal delivery of twins at University of Nigeria Teaching Hospital, Enugu. Niger J Clin Pract. 2013;16(4):490-495. 